\title{
Cooperative Diversity in a Spectrum Sharing Environment
}

\author{
Tea Won Ban, Bang Chul Jung, Member, KIMICS
}

\begin{abstract}
In this paper, we investigate cooperative diversity in a spectrum sharing environment where secondary users utilize primary users' spectrum only if the interference power received at the primary users is maintained below a predetermined level. The outage probability of a selective decode-and-forward (DF) based cooperative diversity scheme in the secondary network is derived to analyze the effects of spectrum sharing on cooperative diversity. Our analytical and simulation results show that the outage probability is saturated at a certain level of transmit power of secondary users due to interference regulation, and, hence, cooperative diversity gains are lost. Through asymptotic analysis, we also identify the critical value of transmit SNR beyond which the outage probability is saturated.
\end{abstract}

Index Terms - congnitive radio, cooperative relaying, diversity, outage probability.

\section{INTRODUCTION}

WITH spectrum sharing techniques, cooperative diversity has been recognized as an effective alternative to the conventional diversity techniques due to a significant gain through cooperation among nodes $[1-8,10,11]$. The concept of cooperative communications originated from a relay channel in information theory [1]. Cooperative transmission between two users was studied in $[2,3]$. Laneman et al. [10] proposed low-complexity cooperative diversity protocols and showed that they achieve full diversity order. Coded cooperation for higher data-rate was studied in $[4,5]$, where a relay node transmits part of a source codeword instead of repeating the source message. Cooperation techniques using multiple relay nodes have been proposed and studied since multiple relays can significantly increase the cooperative diversity gains $[6-8,11]$.

Despite many previous studies on cooperative diversity techniques and spectrum sharing systems, the effects of spectrum sharing on cooperative diversity gains have not been fully investigated yet. Thus, we analyze the key effects of spectrum sharing on cooperative diversity gains

\footnotetext{
Manuscript received August 2, 2011; revised August 29, 2011; accepted September 5, 2011

Tea-Won Ban is with the Mobile laboratory, KT, Seoul, Korea

Bang-Chul Jung (Corresponding Author) is with the Dept. of Information and Communication Engineering, College of Marine Science and Institute of Marine Industry, Gyeongsang National University, Tongyeong, Korea (Email: bcjung@gnu.ac.kr)
}

in terms of outage probability for cooperative communication systems employing spectrum sharing techniques. Our analysis and simulation results will show that the cooperative diversity gains known in a nonspectrum sharing environment are rather lost in the high transmit signal-to-noise (SNR) region due to interference temperature regulation. The rest of this paper is organized as follows: In Section 2, a decode-and-forward (DF)based cooperative system model is described. In Section 3, the outage probabilities of the DF-based cooperative and direct communications in spectrum sharing systems are mathematically analyzed in a slow fading environment. In Section 4, our approximations in asymptotic regions are presented. In Section 5, our discussion is extended to an AF-based cooperative communication system. In Section 6 , numerical results are shown. Finally, conclusions are drawn in Section 7.

\section{SYSTEM AND CHANNEL MODELS}

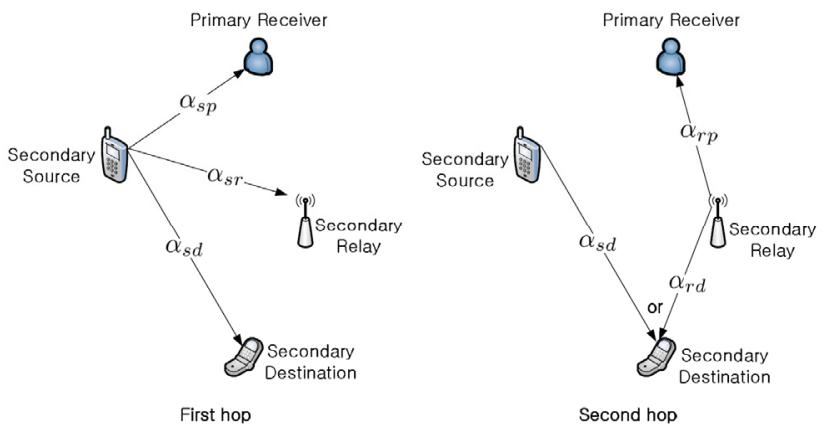

Fig. 1. Cooperative relaying in a spectrum sharing environment

Fig. 1 shows a cooperative relaying system in a spectrum-sharing environment. We consider a repetitionbased two-hop half-duplex cooperative relaying system, which employs a selective decode-and-forward (DF) scheme [10] and shares a spectrum band with a primary receiver. The system consists of a secondary source node, a secondary destination node, a secondary relay node, and a primary receiver denoted by $\mathrm{s}, \mathrm{d}, \mathrm{r}$, and $\mathrm{p}$, respectively. in the first hop, the source node transmits its data to the relay and destination nodes. In the second hop, the relay node forwards the source message to the destination if it successfully decoded the source message. Otherwise, the source node re-transmits its data to the destination node. this cooperative relaying communication is performed 
over two hop periods, the required data rate should be double the data rate of direct communication without relaying to compensate for the spectral efficiency loss due to the transmit duty cycle. Assuming channels between nodes are static over two hop periods, the received signal at node $B$ from node $A$ is represented by

$$
y_{B}=\sqrt{\alpha_{A B}} x_{A}+n_{B},
$$

where $\alpha_{A B}$ denoting the channel gain from node $\mathrm{A}$ to node $\mathrm{B}$ follows an independent and identically distributed (i.i.d.) exponential distribution since we consider independent Rayleigh fading channels and $n_{B}$ denoting the additive white Gaussian noise (AWGN) at node B follows a complex Gaussian distribution. The channel gain is random but remains constant to model a slow fading environment.

When the average transmit power of a secondary source is assumed to be $\mathrm{P}$, the primary user's average received-interference power from the secondary source during a hop period is generally given by

$$
\frac{\int_{0}^{T} P \alpha_{s p}(t) d t}{T},
$$

where $\mathrm{T}$ is the duration of a hop. Since a slow fading environment is assumed, $\alpha_{s p}(t)=\alpha_{s p}$ for T. Then, the average received-interference power can be rewritten as

$$
\frac{P \alpha_{s p} T}{T}=P \alpha_{s p} .
$$

The average interference power perceived at the primary user should be maintained below a given interference level Q. Thus, the effective transmit power of the secondary source is adjusted according to the channel conditions to the primary receiver as follows [9]:

$$
P_{s}^{t x}= \begin{cases}P, & P \alpha_{s p} \leq Q . \\ \frac{Q}{\alpha_{s p}}, & P \alpha_{s p}>Q .\end{cases}
$$

Similarly, the effective transmit power of the secondary relay is obtained as

$$
P_{r}^{t x}= \begin{cases}P, & P \alpha_{s p} \leq Q . \\ \frac{Q}{\alpha_{r p}}, & P \alpha_{s p}>Q .\end{cases}
$$

The relay is assumed to have the same available transmit power, $\mathrm{P}$ for mathematical simplicity. The mean value of channel gains and the variance of white Gaussian noise are assumed to be unity for simplicity of analysis without loss of generality. Correspondingly, the transmit power $\mathrm{P}$ and interference temperature $\mathrm{Q}$ can be also considered as the transmit SNR and interference temperature-to-noise power density, respectively.

\section{ANALYSIS OF OUTAGE PROBABILITY}

TABLE I

OUTAGE EVENTS OF A SELECTIVE DF SYSTEM AND THE CORRESPONDING RECEIVED SNRS

\begin{tabular}{|c|c|c|c|c|}
\hline State & $\begin{array}{c}\text { Source } \\
\text { Tx.power }\end{array}$ & $\begin{array}{c}\text { Relay } \\
\text { Decoding }\end{array}$ & $\begin{array}{c}\text { Relay } \\
\text { Tx.power }\end{array}$ & Received SNR \\
\hline \hline 1 & $P$ & Fail & - & $\gamma_{1}^{D F}=2 P \alpha_{s d}$ \\
\hline 2 & $P$ & Success & $P$ & $\gamma_{2}^{D F}=P\left(\alpha_{s d}+\alpha_{r d}\right)$ \\
\hline 3 & $P$ & Success & $\frac{Q}{\alpha_{r p}}$ & $\gamma_{3}^{D F}=P \alpha_{s d}+\frac{Q \alpha_{r d}}{\alpha_{r p}}$ \\
\hline 4 & $\frac{Q}{\alpha_{s p}}$ & Fail & - & $\gamma_{4}^{D F}=\frac{2 Q \alpha_{s d}}{\alpha_{s p}}$ \\
\hline 5 & $\frac{Q}{\alpha_{s p}}$ & Success & $P$ & $\gamma_{5}^{D F}=\frac{Q \alpha_{s d}}{\alpha_{s p}}+P \alpha_{r d}$ \\
\hline 6 & $\frac{Q}{\alpha_{s p}}$ & Success & $\frac{Q}{\alpha_{r p}}$ & $\gamma_{6}^{D F}=\frac{Q \alpha_{s d}}{\alpha_{s p}}+\frac{Q \alpha_{r d}}{\alpha_{r p}}$ \\
\hline
\end{tabular}

In this section, we analyze outage probability which is a good performance measure in a slow fading environment. In a spectrum sharing environment, outage events in a DF-based cooperative communication system are classified into 6 cases according to the source transmit power, the decoding status at the secondary relay, and relay transmit power as summarized in Table 1. In the first case, the source transmits its data with its full power since the perceived interference at the primary receiver is lower than the interference temperature, but the relay fails in decoding the signal from the source in the first hop $P \alpha_{s p}$ since

$$
\frac{1}{2} \log _{2}\left(1+P \alpha_{s r}\right)<R
$$

where $\mathrm{R}$ represents the required spectral efficiency in a unit of bps/Hz. Then, the source retransmits the signal in the second hop and, hence, an outage event occurs when

$$
\frac{1}{2} \log _{2}\left(1+r_{1}^{D F}\right)<R
$$

where $r_{1}^{D F}$ denotes the received SNR at the destination in the first case and it is given by $2 P \alpha_{s d}$. Thus, the outage probability for this event is given by

$$
\begin{aligned}
p_{1}^{D F} & =\operatorname{Pr}\left[\left(P \alpha_{s p}<Q\right) \cap\left(\frac{1}{2} \log _{2}\left(1+P \alpha_{s r}\right) \cap\left(\frac{1}{2} \log _{2}\left(1+r_{1}^{D F}\right)<R\right)\right]\right. \\
& =\operatorname{Pr}\left[\left(\alpha_{s p}<\frac{Q}{P}\right) \cap\left(\alpha_{s r}<\frac{2^{2 R}-1}{P}\right) \cap\left(\alpha_{s d}<\frac{2^{2 R}-1}{2 P}\right)\right] \\
& =\left(1-e^{-\frac{Q}{P}}\right)\left(1-e^{-R_{p}}\right)\left(1-e^{-\frac{R_{P}}{2}}\right),
\end{aligned}
$$

where $R_{P}$ is defined as

$$
\frac{2^{2 R}-1}{P}
$$


In the second case, the relay node successfully decodes the signal from the source in the first hop and transmits the source signal to the destination with its full power since $P \alpha_{r p}<Q$. Thus, the outage probability can be obtained as follows:

$$
\begin{aligned}
P_{2}^{D F}= & \operatorname{Pr}\left[\left(P \alpha_{s p}<Q\right) \cap\left(\frac{1}{2} \log _{2}\left(1+P \alpha_{s r}\right) \geq R\right) \cap\left(P \alpha_{r p}<Q\right)\right. \\
& \left.\cap\left(\frac{1}{2} \log _{2}\left(1+r_{2}^{D F}\right)<R\right)\right] \\
= & \operatorname{Pr}\left[\left(\alpha_{s p}<\frac{Q}{P}\right) \cap\left(\alpha_{s p} \geq R_{P}\right) \cap\left(\alpha_{r p}<\frac{Q}{P}\right) \cap\left(\alpha_{s d}+\alpha_{r d}<R_{P}\right)\right] \\
= & \left(1-e^{-\frac{Q}{P}}\right)\left(e^{-R_{P}}\right)\left(1-e^{-\frac{Q}{P}}\right)\left(1-e^{-R_{P}}-R_{P} e^{-R_{P}}\right) \\
= & e^{-R_{P}}\left(1-e^{-\frac{Q}{P}}\right)^{2}\left(1-e^{-R_{P}}-R_{P} e^{-R_{P}}\right) .
\end{aligned}
$$

Similarly, the outage probabilities for the remaining four

$$
\begin{aligned}
P_{3}^{D F}= & \operatorname{Pr}\left[\left(P \alpha_{s p}<Q\right) \cap\left(\frac{1}{2} \log _{2}\left(1+P \alpha_{s r}\right) \geq R\right) \cap\left(P \alpha_{r p} \geq Q\right)\right] \\
& \left.\cap\left(\frac{1}{2} \log _{2}\left(1+r_{3}^{D F}\right)<R\right)\right] \\
= & e^{-R_{P}}\left(1-e^{-\frac{Q}{P}}\right) \operatorname{Pr}\left[\left(\alpha_{r p} \geq \frac{Q}{P}\right) \cap\left(\alpha_{s d}+\frac{Q \alpha_{r d}}{P \alpha_{r d}}<R_{P}\right)\right] \\
= & e^{-R_{P}}\left(1-e^{-\frac{Q}{P}}\right) \int_{x=\frac{Q}{P}}^{\infty} e^{-x} \operatorname{Pr}\left[\alpha_{s d}+\frac{Q \alpha_{r d}}{P \alpha_{r d}}<R_{P}\right] d x \\
= & e^{-\frac{Q}{P}-2 R_{P}}\left(1-e^{-\frac{Q}{P}}\right)\left(e^{R_{P}}-\frac{Q}{P} \ln \left(1+R_{Q}\right)-1\right),
\end{aligned}
$$

where $R_{Q}$ is defined as $\frac{2^{2 R}-1}{Q}$

$$
\begin{aligned}
P_{4}^{D F} & =\operatorname{Pr}\left[\left(P \alpha_{s p} \geq Q\right) \cap\left(\frac{1}{2} \log _{2}\left(1+\frac{Q \alpha_{s r}}{\alpha_{s p}}\right)<R\right) \cap\left(\frac{1}{2} \log _{2}\left(1+r_{4}^{D F}\right)<R\right)\right] \\
& =\int_{x=\frac{Q}{P}}^{\infty} e^{-x}\left(1-e^{-R_{Q} x}\right)\left(1-e^{-\frac{R_{Q} x}{2}}\right) d x \\
& =e^{-\frac{Q}{P}}-\frac{e^{-\left(R_{Q}+1\right) \frac{Q}{P}}}{1+R_{Q}}-\frac{2 e^{-\left(\frac{R_{Q}}{2}+1\right) \frac{Q}{P}}}{2+R_{Q}}+\frac{2 e^{-\left(\frac{3 R_{Q}}{2}+1\right) \frac{Q}{P}}}{2+3 R_{Q}} .
\end{aligned}
$$$$
P_{5}^{D F}=\operatorname{Pr}\left[\left(P \alpha_{s p} \geq Q\right) \cap\left(\frac{1}{2} \log _{2}\left(1+\frac{Q \alpha_{s r}}{\alpha_{s p}}\right) \geq R\right) \cap\left(P \alpha_{r p}<Q\right)\right.
$$$$
\left.\cap\left(\frac{1}{2} \log _{2}\left(1+r_{5}^{D F}\right)<R\right)\right]
$$$$
=\left(1-e^{-\frac{Q}{P}}\right) \int_{\frac{Q}{P}}^{\infty} e^{-x\left(1+R_{Q}\right)}\left(1-\frac{x e^{R_{P}}-\frac{Q}{P} e^{-x R_{Q}}}{x-\frac{Q}{P}}\right) d x
$$$$
=e^{-\frac{Q}{P}-R_{P}}\left(1-e^{-\frac{Q}{P}}\right)\left(\frac{1-e^{-R_{P}}}{1+R_{Q}}+e^{-R_{P}} \frac{Q}{P} \ln \left(\frac{1+R_{Q}}{1+2 R_{Q}}\right)\right) .
$$

$$
\begin{aligned}
P_{6}^{D F}= & \operatorname{Pr}\left[\left(P \alpha_{s p} \geq Q\right) \cap\left(\frac{1}{2} \log _{2}\left(1+\frac{Q \alpha_{s r}}{\alpha_{s p}}\right) \geq R\right) \cap\left(P \alpha_{r p} \geq Q\right)\right. \\
& \left.\cap\left(\frac{1}{2} \log _{2}\left(1+r_{6}^{D F}\right)<R\right)\right] \\
= & \int_{\frac{Q}{P}}^{\infty} e^{-x} e^{-R_{Q} x} \frac{\varrho_{\underline{Q}}}{P} e^{-y}\left(1-\frac{x e^{-R_{Q} y}}{x-y}-\frac{x e^{-R_{Q} x}}{x-y}\right) d y d x \\
= & \frac{e^{\frac{-Q}{P}\left(2+R_{Q}\right)}}{1+R_{Q}}-\frac{e^{\frac{-2 Q}{P}\left(1+R_{Q}\right)}}{1+2 R_{Q}} \\
& +\int_{\frac{Q}{P}}^{\infty} x e^{-\left(1+R_{Q}\right) x} \int_{\frac{Q}{P}}^{\infty} \frac{e^{-\left(1+R_{Q}\right) y}}{y-x}-e^{-R_{Q} x} \frac{e^{-y}}{y-x} d y d x .
\end{aligned}
$$

Finally, the overall outage probability of this selective DF-based cooperative communication in the spectrum sharing environment becomes the sum of the outage probabilities of six outage events:

$$
P_{\text {out }}^{D F}=\sum_{i=1}^{6} P_{i}
$$

On the other hand, the outage probability of a direct communication system without a relay in the spectrum sharing environment is obtained as

$$
\begin{aligned}
P_{o u t}^{D}= & \operatorname{Pr}\left[\left(P \alpha_{s p}<Q\right) \cap\left(\log _{2}\left(1+P \alpha_{s d}\right)<R\right)\right] \\
& +\operatorname{Pr}\left[\left(P \alpha_{s p} \geq Q\right) \cap\left(\log _{2}\left(1+\frac{Q \alpha_{s d}}{\alpha_{s p}}\right)<R\right)\right] \\
= & \left(1-e^{-\frac{Q}{P}}\right)\left(1-e^{-\frac{2^{R}-1}{P}}\right)+e^{-\frac{Q}{P}}-\frac{Q}{Q+2^{R}-1} e^{-\frac{\left(Q+2^{R}-1\right)}{P}} .
\end{aligned}
$$

\section{ANALYSIS OF OUTAGE PROBABILITY}

Even though the outage probability in Section 3 can be numerically evaluated, it is not sufficient to intuitively understand the effects of spectrum sharing on cooperative diversity gains. Therefore, we adopt asymptotic analysis to effectively capture the key characteristics of cooperative diversity gains in the spectrum sharing environment.

\section{A. Low Transmit Power}

When the transmit SNR P is asymptotically low, both the secondary source and relay transmit the signal with their full power since $\operatorname{Pr}\left[\alpha_{s p} \leq \frac{Q}{P}\right] \approx 1$ and $\operatorname{Pr}\left[\alpha_{r p} \leq \frac{Q}{P}\right] \approx 1$ Then, the outage probability of the cooperative communication system in the spectrum sharing environment in Eq. (10) is approximated as 


$$
\begin{aligned}
P_{\text {out }}^{D F} \approx & P_{\text {out }, \text { low }}^{D F} \\
= & P_{1}+P_{2} \\
\approx & \operatorname{Pr}\left[\left(\alpha_{s r}<\frac{2^{2 R}-1}{P}\right) \cap\left(\alpha_{s d}<\frac{2^{2 R}-1}{2 P}\right)\right] \\
& +\operatorname{Pr}\left[\left(\alpha_{s r} \geq \frac{2^{2 R}-1}{P}\right) \cap\left(\alpha_{s d}+\alpha_{r d}<\frac{2^{2 R}-1}{2 P}\right)\right] \\
= & \left(1-e^{-R_{P}}\right)\left(1-e^{-\frac{R_{P}}{2}}\right)+e^{-R_{P}}\left(1-e^{-R_{P}}-R_{P} e^{-R_{P}}\right) \\
= & 1-e^{-\frac{R_{P}}{2}}+e^{-\frac{3 R_{P}}{2}}-e^{-2 R_{P}}\left(1+R_{P}\right) .
\end{aligned}
$$

On the other hand, the outage probability of the direct communication system in the spectrum sharing environment in Eq. (11) when the transmit power is sufficiently low is approximated as

$$
P_{\text {out }}^{D F} \approx P_{\text {out }, \text { low }}^{D F}=\operatorname{Pr}\left[\alpha_{\text {sd }}<\frac{2^{R}-1}{P}\right]=1-e^{-\frac{2^{R}-1}{P}}
$$

\section{B. High Transmit Power}

In the high transmit power region, it is obvious that $\operatorname{Pr}\left[\alpha_{s p}>\frac{Q}{P}\right] \approx 1$ and $\operatorname{Pr}\left[\alpha_{r p}>\frac{Q}{P}\right] \approx 1$. Therefore, the outage probability of the cooperative diversity scheme in Eq. (10) is approximated as

$$
P_{\text {out }}^{D F} \approx P_{\text {out }, \text { high }}^{D F}=P_{4}+P_{6}
$$

Using the fact that ${ }^{-x} \approx 1$ for $\ll 1, p_{4}$ in Eq. (7) is approximated as

$$
\begin{aligned}
P_{4}^{D F} & \approx 1-\frac{1}{1+R_{Q}}-\frac{2}{2+R_{Q}}+\frac{2}{2+3 R_{Q}} \\
& =\frac{R_{Q}^{2}\left(4+3 R_{Q}\right)}{\left(1+R_{Q}\right)\left(2+R_{Q}\right)\left(2+3 R_{Q}\right)}
\end{aligned}
$$

and $P_{6}^{D F}$ in Eq. (9) is approximated as

$$
\begin{aligned}
P_{6}^{D F} & \approx \operatorname{Pr}\left[\left(\frac{\alpha_{s r}}{\alpha_{s p}} \geq R_{Q}\right) \cap\left(\frac{\alpha_{s d}}{\alpha_{s p}}+\frac{\alpha_{r d}}{\alpha_{r p}}<R_{Q}\right)\right] \\
& =\int_{0}^{\infty} \operatorname{Pr}\left[\alpha_{s p}=x\right] \operatorname{Pr}\left[\alpha_{s r}>R_{Q} x\right] \operatorname{Pr}\left[\frac{\alpha_{s d}}{x}+\frac{\alpha_{r d}}{\alpha_{r p}}<R_{Q}\right] d x \\
& =\int_{0}^{\infty} e^{-\left(R_{Q}+1\right) x} \operatorname{Pr}\left[\frac{\alpha_{s d}}{x}+\frac{\alpha_{r d}}{\alpha_{r p}}<R_{Q}\right] d x .
\end{aligned}
$$

Let ${ }_{Y=\frac{\alpha_{s d}}{x}}$ and $Z=\frac{\alpha_{r d}}{\alpha_{r p}}$, then, $\operatorname{Pr}\left[\frac{\alpha_{s d}}{x}+\frac{\alpha_{r d}}{\alpha_{r p}}<R_{Q}\right]$ in Eq. (16) is given by

$$
\begin{aligned}
\operatorname{Pr}\left[\frac{\alpha_{s d}}{x}+\frac{\alpha_{r d}}{\alpha_{r p}}<R_{Q}\right] & =\int_{0}^{R_{Q}} f Y(y) \int_{0}^{R_{Q}-y} f Z(z) d z d y \\
& =\int_{0}^{R_{Q}} x e^{-x y} F_{Z}\left(R_{Q}-y\right) d y \\
& =1-e^{-R_{Q} x}+x e^{-\left(R_{Q}+1\right) x}\left(E_{i}\left(-\left(R_{Q}+1\right) x\right)-E_{i}(-x)\right)
\end{aligned}
$$

where $\mathrm{F}_{Z}(\mathrm{z})$ is the $\mathrm{CDF}$ of the ratio of two i.i.d. exponentially distributed random variables given by [12]

$$
F_{Z}(z)=\frac{z}{1+z}
$$

and $E_{i}(\cdot)$ represents the exponential integral function. Substituting Eq. (17) into Eq. (16) yields the outage probability.

$$
\begin{aligned}
P_{6}^{D F} & \approx \int_{0}^{\infty} e^{-\left(R_{Q}+1\right) x}-e^{-\left(2 R_{Q}+1\right) x}+x e^{-2\left(R_{Q}+1\right) x}\left(E_{i}\left(-\left(R_{Q}+1\right) x\right)-E_{i}(-x)\right) d x \\
& =\frac{1}{R_{Q}+1}-\frac{1}{2 R_{Q}+1}+\left(\frac{j \pi-\ln \left[-\left(1+2 R_{Q}\right)\right]}{4\left(R_{Q}+1\right)^{2}}-\frac{R_{Q}}{2\left(R_{Q}+1\right)^{2}\left(2 R_{Q}+1\right)}\right) \\
& =\frac{2 R_{Q}-\ln \left(1+2 R_{Q}\right)}{4\left(R_{Q}+1\right)^{2}},
\end{aligned}
$$

where $\mathrm{j}$ denotes $\sqrt{-1}$ and $\ln (-x)=j \pi+\ln \mathrm{x}$ for $\mathrm{x}>0$. From Eq. (15) and Eq. (19), the outage probability in the high transmit SNR region, $P_{\text {out }, \text { high }}^{D F}$, is obtained as

$$
P_{\text {out } \text {, high }}^{D F}=\frac{R_{Q}^{2}\left(4+3 R_{Q}\right)}{\left(1+R_{Q}\right)\left(2+R_{Q}\right)\left(2+3 R_{Q}\right)}+\frac{2 R_{Q}-\ln \left(2 R_{Q}+1\right)}{4\left(R_{Q}+1\right)^{2}}
$$

Note that the outage probability in Eq. (20) is not a function of $\mathrm{P}$ since $R_{Q}=\frac{2^{2 R}-1}{Q}$. Therefore, the outage probability is predicted to be saturated as the transmit SNR P becomes larger, which will be confirmed later in numerical results.

On the other hand, $P_{\text {out }}^{D}$ in Eq. (11) is approximated for the high transmit power as

$$
\begin{aligned}
P_{o u t}^{D} & \approx P_{\text {out }, \text { high }}^{D} \\
& \left.=\operatorname{Pr}\left[\log _{2}\left(1+\frac{Q \alpha_{s d}}{\alpha_{s p}}\right)<R\right)\right] \\
& =\operatorname{Pr}\left[\frac{\alpha_{s d}}{\alpha_{s p}}<\frac{2^{R}-1}{Q}\right] \\
& =\frac{2^{R}-1}{2^{R}-1+Q} .
\end{aligned}
$$

\section{EXTENSION TO AF-BASED COOPERATIVE COMMUNICATION}

In this section, we extend our discussion to an AFbased cooperative communication system in a spectrum sharing environment. The received SNR at the destination node in AF-based cooperative relaying systems is obtained as [10]

$$
r^{A F} \approx P_{s} \alpha_{s d}+\frac{P_{s} \alpha_{r d} \alpha_{s r}}{\alpha_{r d}+\frac{1}{\beta^{2}}}
$$


where $P_{s}$ denotes the transmit power of a source in secondary networks and $\beta$ denotes the amplification gain of a relay in the secondary networks given by

$$
\beta^{2}=\frac{P_{r}}{P_{s} \alpha_{s r}+1}
$$

where $P_{r}$ denotes the transmit power of a relay in secondary networks. Contrary to the selective DF scheme, an AF-based relay node amplifies and forwards its received signal without decoding the signal from the source node. Thus, the outage events of the AF-based cooperative diversity scheme in the spectrum sharing environment are classified into the following four cases according to the source transmit power and relay transmit power as summarized in Table 2. The corresponding received SNRs of the four outage events are summarized

TABLE I I

OUTAGE EVENTS OF THE AF SYSTEM AND THE CORRESPONDING RECEIVED SNRS

\begin{tabular}{|c|c|c|c|l|}
\hline State & $\begin{array}{c}\text { Source } \\
\text { power }\end{array}$ & $\begin{array}{c}\text { Relay } \\
\text { power }\end{array}$ & $\begin{array}{c}\text { Relay } \\
\text { Amp. Gain }\end{array}$ & \multicolumn{1}{|c|}{ Received SNR } \\
\hline \hline 1 & $P$ & $P$ & $\beta=\sqrt{\frac{P}{P \alpha_{s r}+1}}$ & $\gamma_{1}^{A F}=P \alpha_{s d}+\frac{P^{2} \alpha_{s r} \alpha_{r d}}{P \alpha_{s r}+P \alpha_{r d}+1}$ \\
\hline 2 & $P$ & $\frac{Q}{\alpha_{r p}}$ & $\beta=\sqrt{\frac{Q}{\left(P \alpha_{s r}+1\right) \alpha_{r p}}}$ & $\gamma_{2}^{A F}=P \alpha_{s d}+\frac{P Q \alpha_{s r} \alpha_{r d}}{Q \alpha_{r d}+P \alpha_{s r} \alpha_{r p}+\alpha_{r p}}$ \\
\hline 3 & $\frac{Q}{\alpha_{s p}}$ & $P$ & $\beta=\sqrt{\frac{P}{\frac{Q \alpha_{s r}}{\alpha_{s p}}+1}}$ & $\gamma_{3}^{A F}=\frac{Q \alpha_{s d}}{\alpha_{s p}}+\frac{P Q \alpha_{s r} \alpha_{r d}}{P \alpha_{r d} \alpha_{s p}+Q \alpha_{s r}+\alpha_{s p}}$ \\
\hline 4 & $\frac{Q}{\alpha_{s p}}$ & $\frac{Q}{\alpha_{r p}}$ & $\beta=\sqrt{\frac{Q}{\left(\frac{Q \alpha_{s r}}{\alpha_{s p}}+1\right) \alpha_{r p}}}$ & $\gamma_{4}^{A F}=\frac{Q \alpha_{s d}}{\alpha_{s p}}+\frac{Q^{2} \alpha_{s r} \alpha_{r d}}{Q \alpha_{r d} \alpha_{s p}+Q \alpha_{s r} \alpha_{r p}+\alpha_{s p} \alpha_{r p}}$ \\
\hline
\end{tabular}

in Table 2. However, since the closed-form statistical distributions of the received SNRs are not known, mathematical analysis for the outage probability is intractable $[10,13]$. Thus, for analytical tractability, we assume that the noise power in Eq. (23) is negligible. This assumption can be justified when the noise power is relatively small compared to the received signal power [13]. Based on this assumption, the amplification gain at each relay node in Eq. (23) is approximated as

$$
\beta^{2} \approx \frac{P_{r}}{P_{s} \alpha_{s r}}
$$

using the amplification gain in Eq. (24), we derive the statistical distributions of the received SNRs in the last column of Table 2 and compute the outage probability.

According to Table 2, the first outage event occurs with the probability given by

$$
\begin{aligned}
P_{1}^{A F} & =\operatorname{Pr}\left[\left(P \alpha_{s p}<Q\right) \cap\left(P \alpha_{r p}<Q\right) \cap\left(\frac{1}{2} \log _{2}\left(1+r_{1}^{A F}\right)<R\right)\right] \\
& \approx \operatorname{Pr}\left[\left(\alpha_{s p}<\frac{Q}{P}\right) \cap\left(\alpha_{r p}<\frac{Q}{P}\right) \cap\left(\alpha_{s d}+\frac{\alpha_{s r} \alpha_{r d}}{\alpha_{s r}+\alpha_{r d}}<R_{P}\right)\right] \\
& =\left(1-e^{-\frac{Q}{P}}\right)^{2} \operatorname{Pr}\left[\alpha_{s d}+\frac{\alpha_{s r} \alpha_{r d}}{\alpha_{s r}+\alpha_{r d}}<R_{P}\right] .
\end{aligned}
$$

Let $X=\alpha_{s d}$ and $Y=\frac{2 \alpha_{s r} \alpha_{r d}}{\alpha_{s r}+\alpha_{r d}}$. Then, $\mathrm{Y}$ is the harmonic mean of two independent exponential random variables whose statistical distribution is obtained by using the following proposition: Proposition 6.5.1 (CDF of a harmonic mean [13]) The harmonic mean of two random variables $\mathrm{Y} 1$ and Y2 is defined as

$$
Y=\frac{2 Y_{1} Y_{2}}{Y_{1}+Y_{2}}
$$

If $\mathrm{Y} 1$ and $\mathrm{Y} 2$ be independent exponentially distributed random variables with means $\beta_{1}$ and $\beta_{2}$ respectively, the $\mathrm{CDF}$ of $\mathrm{Y}$ is given by

$$
F_{Y\left|\left(\beta_{1}, \beta_{2}\right)\right|}(y)=1-\frac{y}{\sqrt{\beta_{1} \beta_{2}}} e^{-\frac{y\left(\beta_{1}+\beta_{2}\right)}{2 \beta_{1} \beta_{2}}} K_{1}\left(\frac{y}{\sqrt{\beta_{1} \beta_{2}}}\right)
$$

where $\mathrm{K} 1(\cdot)$ is the first-order modified Bessel function of the second kind.

Using Proposition 1, Eq. (25) is given by

$$
\begin{aligned}
P_{1}^{A F} & =\left(1-e^{-\frac{Q}{P}}\right)^{2} \operatorname{Pr}\left[X+\frac{Y}{2}<R_{P}\right] \\
& =\left(1-e^{-\frac{Q}{P}}\right)^{2} \int_{0}^{R_{P}} e^{-x} \int_{0}^{2\left(R_{P}-x\right)} f_{Y \mid(1,1)}(y) d y d x \\
& =\left(1-e^{-\frac{Q}{P}}\right)^{2} \int_{0}^{R_{P}} e^{-x} F_{Y \mid(1,1)}\left(2\left(R_{P}-x\right)\right) d x .
\end{aligned}
$$

Similarly, the second outage event in Table 2 occurs with probability given by

$$
\begin{aligned}
P_{2}^{A F} & =\operatorname{Pr}\left[\left(P \alpha_{s p}<Q\right) \cap\left(P \alpha_{s p} \geq Q\right) \cap\left(\frac{1}{2} \log _{2}\left(1+r_{2}^{A F}\right)<R\right)\right] \\
& \approx \operatorname{Pr}\left[\left(\alpha_{s p}<\frac{Q}{P}\right) \cap\left(\alpha_{r p} \geq \frac{Q}{P}\right) \cap\left(\alpha_{s d}+\frac{Q \alpha_{s r} \alpha_{r d}}{Q \alpha_{r d}+P \alpha_{s r} \alpha_{r p}}<R_{P}\right)\right] \\
& =\left(1-e^{-\frac{Q}{P}}\right) \operatorname{Pr}\left[\left(\alpha_{r p} \geq \frac{Q}{P}\right) \cap\left(\alpha_{s d}+\frac{Q \alpha_{s r} \alpha_{r d}}{Q \alpha_{r d}+P \alpha_{s r} \alpha_{r p}}<R_{P}\right)\right] \\
& =\left(1-e^{-\frac{Q}{P}}\right) \int_{\omega=\frac{Q}{P}}^{\infty} e^{-\omega} \operatorname{Pr}\left[X+\frac{Y}{2 \omega P}<R_{P}\right] d \omega
\end{aligned}
$$

where $X=\alpha_{s d}$ and $Y=\frac{2 P Q w \alpha_{s r} \alpha_{r d}}{Q \alpha_{r d}+w P \alpha_{s r}}$. It should be noted here that $\mathrm{Y}$ conditioned on $\mathrm{W}$ is the harmonic mean of two independent exponential random variables $Q \alpha_{r d}$ and $w P \alpha_{s r}$ with mean $\mathrm{Q}$ and $w P$, respectively. From Proposition 1, the probability in Eq. (29) is given by

$$
\begin{aligned}
P_{2}^{A F} & =\left(1-e^{-\frac{Q}{P}}\right) \int_{\omega=\frac{Q}{P}}^{\infty} e^{-\omega} \int_{x=0}^{R_{P}} e^{-x} \int_{0}^{2 \omega P\left(R_{P}-x\right)} f_{Y(Q, \omega P)}(y) d y d x d \omega \\
& =\left(1-e^{-\frac{Q}{P}}\right) \int_{\omega=\frac{Q}{P}}^{\infty} e^{-\omega} \int_{x=0}^{R_{P}} e^{-x} F_{Y((Q, \omega P))}\left(2 \omega P\left(R_{P}-x\right)\right) d x d \omega
\end{aligned}
$$


The probability that the third outage event in Table 2 occurs is given by

$$
\begin{aligned}
P_{3}^{A F} & =\operatorname{Pr}\left[\left(P \alpha_{s p} \geq Q\right) \cap\left(P \alpha_{r p}<Q\right) \cap\left(\frac{1}{2} \log _{2}\left(1+r_{3}^{A F}\right)<R\right)\right] \\
& \approx \operatorname{Pr}\left[\left(\alpha_{s p} \geq \frac{Q}{P}\right) \cap\left(\alpha_{r p}<\frac{Q}{P}\right) \cap\left(\frac{\alpha_{s d}}{\alpha_{s p}}+\frac{P \alpha_{s r} \alpha_{r d}}{P \alpha_{r d} \alpha_{s p}+Q \alpha_{s r}}<R_{Q}\right)\right] \\
& =\left(1-e^{-\frac{Q}{P}}\right) \operatorname{Pr}\left[\left(\alpha_{s p} \geq \frac{Q}{P}\right) \cap\left(\frac{\alpha_{s d}}{\alpha_{s p}}+\frac{P \alpha_{s r} \alpha_{r d}}{P \alpha_{r d} \alpha_{s p}+Q \alpha_{s r}}<R_{Q}\right)\right] \\
& =\left(1-e^{-\frac{Q}{P}}\right) \int_{\frac{Q}{P}}^{\infty} e^{-\omega} \operatorname{Pr}\left[X+\frac{Y}{2 Q \omega}<R_{Q}\right] d \omega
\end{aligned}
$$

where $X=\frac{\alpha_{s d}}{w}$ and $Y=\frac{2 P Q w \alpha_{s r} \alpha_{r d}}{P w \alpha_{r d}+Q \alpha_{s r}}$. Conditioned on $\mathrm{w}, \mathrm{X}$ is an exponentially distributed random variable with mean $1 / \mathrm{w}$ and $\mathrm{Y}$ is the harmonic mean of two exponentially distributed random variables $P w \alpha_{r d}$ and $Q \alpha_{s r}$ the probability of the third outage event in Eq. (31) is obtained as

$$
\begin{aligned}
P_{3}^{A F} & =\left(1-e^{-\frac{Q}{P}}\right) \int_{\omega=\frac{Q}{P}}^{\infty} e^{-\omega} \int_{x=0}^{R_{Q}} \omega e^{-\omega x} \int_{0}^{2 Q \omega\left(R_{Q}-x\right)} f_{Y|(Q, P \omega)|}(y) d y d x d \omega \\
& =\left(1-e^{-\frac{Q}{P}}\right) \int_{\omega=\frac{Q}{P}}^{\infty} e^{-\omega} \int_{x=0}^{R_{Q}} \omega e^{-\omega x} F_{Y|(Q, P \omega)|}\left(2 Q \omega\left(R_{Q}-x\right)\right) d x d \omega .
\end{aligned}
$$

Finally, the probability corresponding to the fourth outage event in Table 2 is given by

$$
\begin{aligned}
P_{4}^{A F} & =\operatorname{Pr}\left[\left(P \alpha_{s p} \geq Q\right) \cap\left(P \alpha_{r p} \geq Q\right) \cap\left(\frac{1}{2} \log _{2}\left(1+r_{4}^{A F}\right)<R\right)\right] \\
& \approx \operatorname{Pr}\left[\left(\alpha_{s p} \geq \frac{Q}{P}\right) \cap\left(\alpha_{r p} \geq \frac{Q}{P}\right) \cap\left(\frac{\alpha_{s d}}{\alpha_{s p}}+\frac{\alpha_{s r} \alpha_{r d}}{\alpha_{r d} \alpha_{s p}+\alpha_{s r} \alpha_{r p}}<R_{Q}\right)\right] \\
& =\int_{\frac{Q}{P}}^{\infty} e^{-\omega} \int_{\frac{Q}{P}}^{\infty} e^{-z} \operatorname{Pr}\left[X+\frac{Y}{2 \omega z}<R_{Q}\right] d z d \omega
\end{aligned}
$$

where $X=\frac{\alpha_{s d}}{w}$ and $Y=\frac{2 w z \alpha_{s r} \alpha_{r d}}{w \alpha_{s r}+z \alpha_{r d}}$. Conditioned on $\mathrm{w}$ and $\mathrm{z}$, $\mathrm{X}$ follows an exponential distribution with mean $1 / \mathrm{w}$ and $\mathrm{Y}$ is the harmonic mean of two independent exponentially distributed random variables $w \alpha_{s r}$ and $z \alpha_{r d}$. Correspondingly, Eq. (33) is derived from Proposition 1 as

$$
\begin{aligned}
P_{4}^{A F} & =\int_{\frac{Q}{P}}^{\infty} e^{-\omega} \int_{\frac{Q}{P}}^{R_{P}} e^{-z} \int_{0}^{R_{Q}} \omega e^{-\omega x} \int_{0}^{2 \omega z\left(R_{Q}-x\right)} f_{Y|(w, z)|}(y) d y d x d z d \omega \\
& =\int_{\frac{Q}{P}}^{\infty} e^{-\omega} \int_{\frac{Q}{P}}^{R_{P}} e^{-z} \int_{0}^{R_{Q}} \omega e^{-\omega x} F_{Y|(\omega, z)|}\left(2 \omega z\left(R_{Q}-x\right) d x d z d \omega .\right.
\end{aligned}
$$

Then, the outage probability of the AF-based cooperative communication system in the spectrum sharing environment is obtained as

$$
P_{\text {out }}^{A F}=\sum_{i=1}^{4} P_{i}^{A F}
$$

\section{NUMERICAL RESULTS}

This section presents numerical results about the effects of spectrum sharing on cooperative diversity gains. We assume that the required spectral efficiency $\mathrm{R}$ is set to 0.1 $\mathrm{bps} / \mathrm{Hz}$ in the following results.

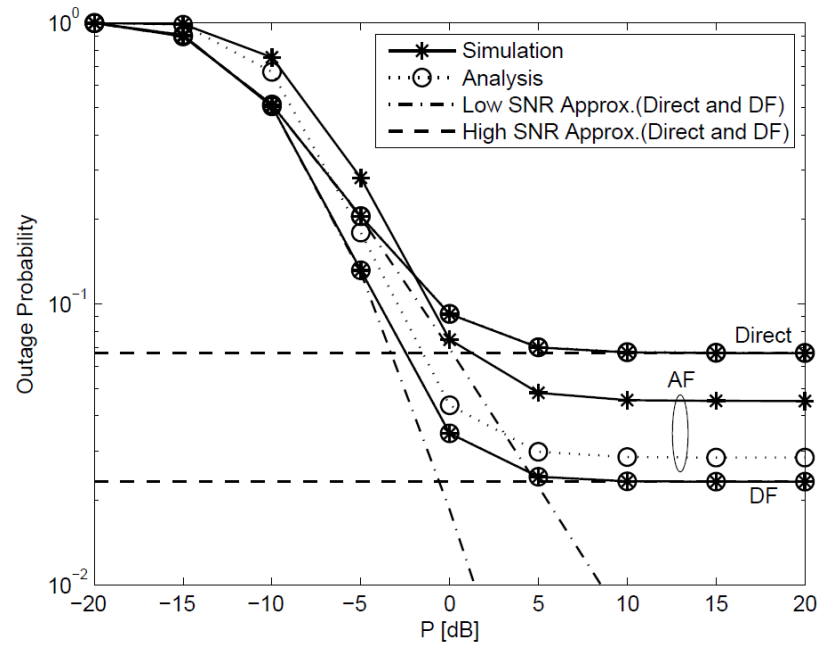

(a) $Q=0 d B$

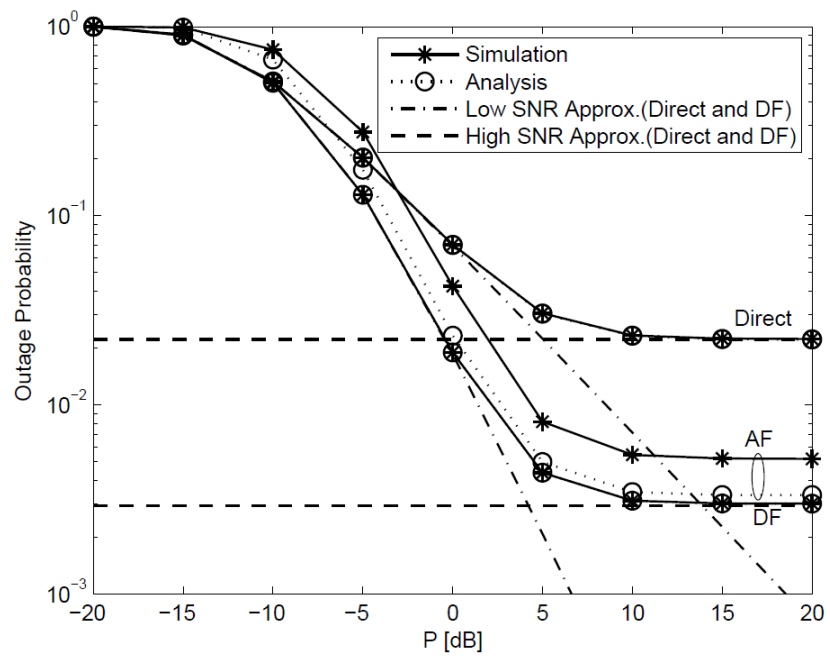

(b) $Q=5 d B$

Fig. 2. Outage probability versus the transmit SNR. $\mathrm{R}=0.1 \mathrm{bps} / \mathrm{Hz}$

Fig. 2 shows the outage probabilities versus the transmit SNR P when the interference temperature $\mathrm{Q}$ is set to $0 \mathrm{~dB}$ or $5 \mathrm{~dB}$. It is shown that the outage probabilities of all the schemes become saturated beyond a certain level of $\mathrm{P}$ due to the interference regulation. As the interference regulation becomes tighter (smaller Q), the saturation occurs in the lower transmit SNR region. These results indicate that 
cooperative diversity gains are lost in the high transmit SNR region unlike in non-spectrum sharing environments. This also shows that we can estimate the saturated value of outage probability and the corresponding transmit SNR value through our asymptotic analysis in the high and low SNR regions. In addition, we can characterize the outage performance through this asymptotic analysis even before the saturation occurs. There exists a difference between the outage probabilities of both the AF-based cooperative communications and DF-based cooperative communications in the high SNR region, while the difference disappears in the high SNR region in nonspectrum sharing environments. For the DF-based cooperative communication scheme, we confirm that the analytical results agree well with simulation results regardless of the values of $\mathrm{Q}$. On the other hand, for the AF-based cooperative communication scheme, the analytical approximation result yields lower bounds compared with the simulation result. However, this bound becomes tighter as Q increases because the effect of noise power can be neglected due to higher transmit power under less tight interference regulation.

As shown in Fig.2, the outage probability becomes saturated beyond a certain value of $\mathrm{P}$ and, hence, the cooperative diversity gains promised in the non-spectrum sharing environment are lost. The saturation value of transmit SNR, $P_{\text {sat }}$ can be determined through the asymptotic results. $P_{\text {sat }}$ is obtained as the point where two asymptotic curves intersect. Fig. 3 shows the saturation values of transmit SNR, $P_{\text {sat }}$, of both the DF and direct transmission schemes for varying $Q$ values. $P_{\text {sat }}$ linearly increases in $\mathrm{dB}$ scale with $\mathrm{Q}$ values because larger transmit power of secondary users is allowed as $\mathrm{Q}$ increases.

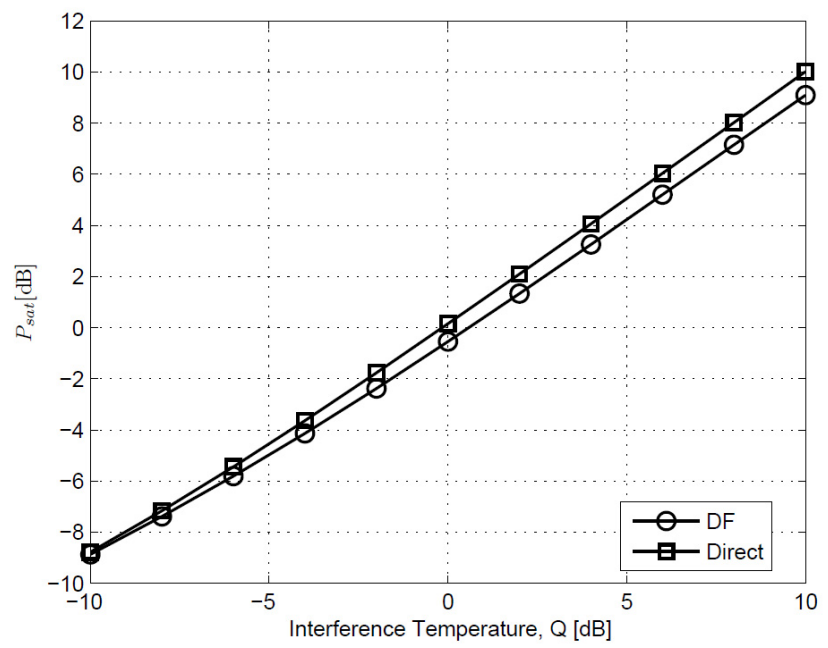

Fig. 3. Transmit SNR where the outage probability is saturated. $\mathrm{R}=0.1 \mathrm{bps} / \mathrm{Hz}$

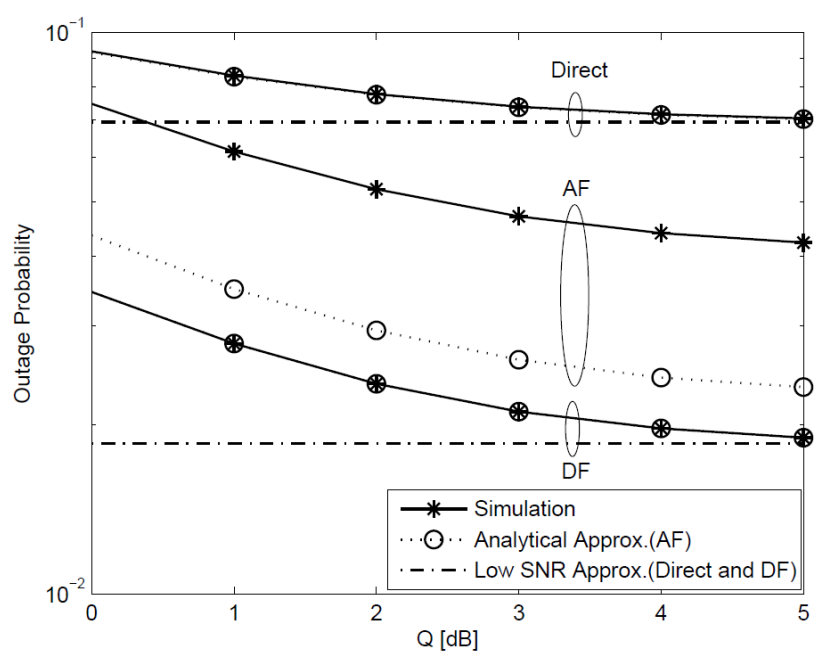

(a) $\mathrm{P}=0 \mathrm{~dB}$

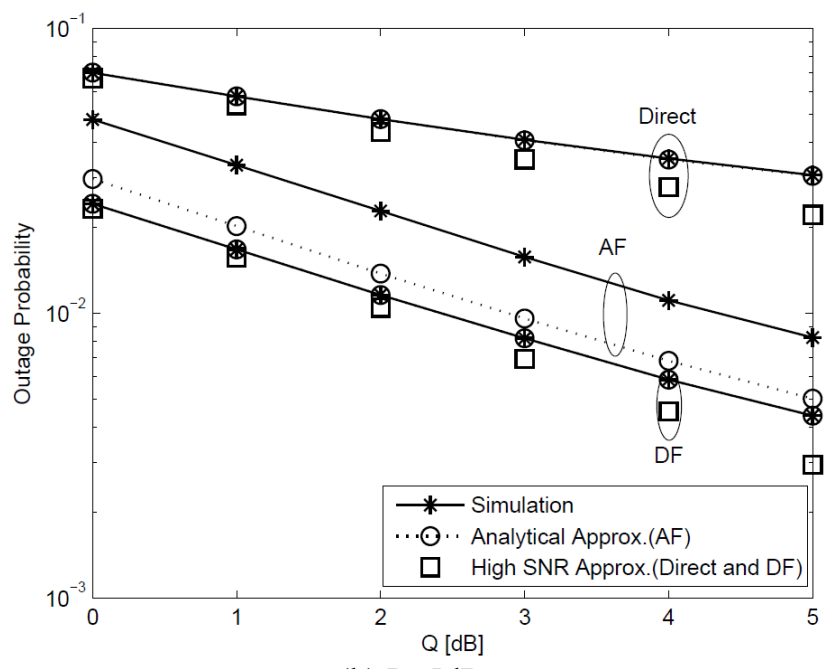

(b) $\mathrm{P}=5 \mathrm{~dB}$

Fig. 4. Outage probability versus the interference temperature. $\mathrm{R}=0.1 \mathrm{bps} / \mathrm{Hz}$

Fig. 4 shows the outage probabilities versus $\mathrm{Q}$ when $\mathrm{P}$ is set to 0 or $5 \mathrm{~dB}$. It is shown that the outage probabilities for all schemes decrease as Q increases because the increasing $\mathrm{Q}$ relaxes the interference regulation and, hence, increases the effective transmit power of the source and relay in the secondary networks. It is again confirmed that the analytical results agree with simulation results very well for the direct and DF-based communication schemes. The analytical approximation results of the AFbased cooperative communication scheme approach the simulation results as $\mathrm{P}$ increases.

\section{CONCLUSIONS}

In this paper, we analyzed cooperative diversity gains in terms of outage probability in a spectrum sharing environment where the transmit power of source and relay 
is regulated for a given interference temperature at a primary user. Our analytical and simulation results showed that the cooperative diversity gains promised in non-spectrum sharing environments are lost beyond a certain value of transmit SNR if there exits an interference regulation for a primary receiver. We can characterize the outage performance through our asymptotic analysis and can also find the critical value of transmit SNR beyond which the outage probability becomes saturated.

\section{ACKNOWLEDGEMENT}

This research was supported by Basic Science Research Program Through the National Research Foundation of Korea(NRF) funded by Ministry of Education, Science and Technology (2010-0011140)

\section{REFERENCES}

[1] T. M. Cover and A. A. E. Gamal, "Capacity theorems for the relay channel," IEEE Trans-actions on Information Theory, vol. 25, no. 5, pp. 572-584, September 1979.

[2] A. Sendonaris, E. Erkip, and B. Aazhang, "User cooperation diversity-Part I: System description," IEEE Transactions on Communications, vol. 51, no. 11, pp. 1927-1938, November 2003.

[3] A. Sendonaris, E. Erkip, and B. Aazhang, "User cooperation diversity-Part II: Implementation aspects and performance analysis," IEEE Transactions on Communications, vol. 51, no. 11, pp. 1939-1948, November 2003.

[4] T. E. Hunter and A. Nosratinia, "Cooperation diversity through coding," Proc. of IEEE ISIT, p. 220, 2002.

[5] M. Janani, A. Hedayat, T. E. Hunter, and A. Nosratinia, "Coded cooperation in wireless communications:space-time transmission and iterative decoding," IEEE Transactions on Signal Processing, vol. 52, no. 2, pp. 362-371, February 2004.

[6] K. G. Seddik, A. K. Sadek, W. Su, and K. J. R. Liu, "Outage analysis of multi-node amplify-and-forward relay networks," Proc. of IEEE WCNC, pp. 1184-1188, 2006.

[7] A. Adinoyi and H. Yanikomeroglu, "Cooperative relaying in multiantenna fixed relay networks," IEEE Transactions on Wireless Communications, vol. 6, no. 2, pp. 533-544, February 2007.

[8] A. Bletsas, A. Khisti, D. P. Reed, and A. Lippman, "A simple cooperative diversity method based on network path selection," IEEE Journal on Selected Areas in Communications, vol. 24, no. 3, pp. 659-672, March 2006.

[9] A. Ghasemi and E. S. Sousa, "Fundamental limits of spectrumsharing in fading environments," IEEE Transactions on Wireless Communications, vol. 6, no. 2, pp. 649-658, February 2007.

[10] J. N. Laneman, D. N. C. Tse, and G.W.Wornell, "Cooperative diversity in wireless networks: Efficient protocols and outage behavior," IEEE Transactions on Information Theory, vol. 50, no. 12, pp. 3062-3080, December 2004.

[11] T. W. Ban, W. Choi, B. C. Jung, and D. K. Sung, "A cooperative phase steering scheme in multi-relay node environments," IEEE Transactions on Wireless Communications, vol. 8, no. 1, pp. 72-77, January 2009.

[12] A. Papoulis and S. U. Pillai, Probability, Random Variables and Stochastic Processes, 4th ed. McGraw-Hill, 2002.

[13] M. O. Hasna and M.-S. Alouini, "End-to-end performance of transmission systems with relays over Rayleigh-fading channels," IEEE Transactions on Wireless Communications, vol. 2, no. 6, pp. 1126-1131, November 2003.

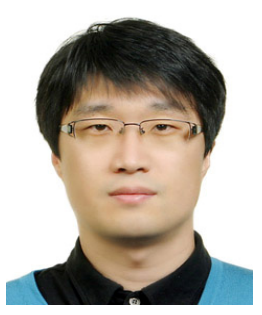

Tae Won Ban received the B.S. and M.S. degrees in electronics engineering from Kyungpook National University, Korea, in 1998 and 2000, respectively, and the Ph.D. degree in electrical engineering from the Korea Advanced Institute of Science and Technology (KAIST), Korea, in 2010. Since January 2000, he has been working for Korea Telecom, Korea. His research interests include OFDM, MIMO, radio resource management for mobile communication systems, cognitive radio, and relay systems.

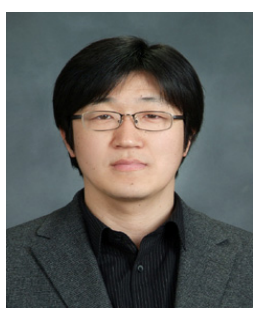

Bang Chul Jung received the B.S. degree in Electronics Engineering from Ajou University, Suwon, Korea, in 2002 and the M.S. and Ph.D degrees in Electrical \& Computer Engineering from Korea Advanced Institute of Science and Technology (KAIST), Daejeon, Korea, in 2004 and 2008, respectively. He was a research professor with KAIST Institute for Information Technology Convergence, Daejeon, Korea, until Feb. 2010. He is now an assistant professor of department of Information and Communication Engineering, Gyeonsang National University, Korea. Dr. Jung is a member of IEEE, IEICE, Marquis Who's Who in the World (2011 Edition), IBC Top 100 Engineers (2011 Edition). He was the recipient of the Bronze Prize in Intel Student Paper Contest in 2005, the First Prize in Research Performance Evaluation Sytem (RPES) for Doctorial Student (School of EECS, KAIST) in 2008, the First Prize in KAIST's Invention Idea Contest in 2008, the Bronze Prize in Samsung Humantech Paper Contest in 2009, the IEEE Communication Society Asia-Pacific Outstanding Young Researcher Award in 2011. 